\title{
Establishment of a Linear Correlation Model of Central Venous Blood Oxygen Saturation and Lactate in Sepsis
}

\author{
Songchang Shi ${ }^{1}$, Xiaobin Pan ${ }^{1}$, Wei Lin ${ }^{2}$, Chao Wu ${ }^{1}$, Mei Ye ${ }^{1}$, Yingfeng Zhuang ${ }^{1}$, \\ Jian Lin ${ }^{1}$, Xincai Wang ${ }^{1}$, Lihui Zhang ${ }^{1}$, Shujuan Zhang ${ }^{1}$, Hangwei Feng ${ }^{1}$, Long Huang ${ }^{1}$, \\ Songjing Shi ${ }^{3, *}$, Xingsheng Lin ${ }^{1, *}$
}

\begin{abstract}
${ }^{1}$ Department of Critical Care Medicine, Shengli Clinical Medical College of Fujian Medical University, Fujian Provincial Hospital South Branch, Fuzhou, 350001, P. R. China

${ }^{2}$ Department of Endocrinolog, Shengli Clinical Medical College of Fujian Medical University, Fujian Provincial Hospital, FuZhou, 350001, P. R. China ${ }^{3}$ Department of Critical Care Medicine, Shengli Clinical Medical College of Fujian Medical University, Fujian Provincial Hospital, Fuzhou, 350001, P. R. China
\end{abstract}

\section{*Correspondence}

songjingshifz@163.com

(Songjing Shi)

xingshenglinfz@163.com

(Xingsheng Lin)

\begin{abstract}
Introduction: Studies have shown that there is a complex relationship between lactate and $\mathrm{ScvO}_{2}$. Methods: A retrospective study was carried out in 37 intensive care patients with sepsis or septic shock. The relationship between lactate and $\mathrm{ScvO} 2$ was explored with correlation analysis and simple linear modelling. Results: Lactate and ScvO2 were significantly correlated in patients with septic shock $(\mathrm{r} 2=0.46, \mathrm{p}=0.001 ; y=-4.11 x$ +82.62 ), but not in sepsis. $y$ Significant correlation between these parameters was also found in the group of patients who went on to die ( $\mathrm{r} 2=0.67, \mathrm{p}<0.01 ; y=-3.70 x+$ 78.61), but not in patients who survived. Conclusions: In sepsis, the correlation between $\mathrm{ScvO}_{2}$ and lactate is not constant over the sepsis course and may be dynamic. In the resuscitation of sepsis and/or septic shock, changes in $\mathrm{ScvO} 2$ requires further study.
\end{abstract}

\section{Keywords}

Central venous blood oxygen saturation, Lactate, Sepsis, Septic shock

\section{Introduction}

Sepsis and septic shock are common and potentially lethal complications of chronic illness and acute organ dysfunction secondary to infection $[1,2]$. Sepsis is a leading cause of mortality and critical illness worldwide $[3,4]$. The incidence of sepsis and septic shock in adults ranges from 56 to 91 per 100,000 population per year [5]. Short term mortality is $20-30 \%$, reaching up to $50 \%$ in patients with septic shock [6]. Despite improvements in sepsis care, there has been neither a significant increase in the incidence of sepsis nor a significant improvement in outcomes between 2009 and 2014 [7]. This is despite efforts to improve methods of identifying and managing sepsis with the aim of reducing mortality [8].

Studies of the critically ill have shown that lactate can be used as a marker of tissue hypoxia [9]. Another marker is central venous oxygen saturation $\left(\mathrm{ScvO}_{2}\right)$, which is a surrogate marker of oxygenation of venous return and hence oxygen delivery and tissue consumption [10]. The relationship between lactate and $\mathrm{ScvO} 2$ has been shown to be complex [11, 12].

As both lactate and $\mathrm{ScvO}_{2}$ are markers of tissue oxygenation [13-16], it was posited that levels of lactate and $\mathrm{ScvO}_{2}$ may be correlated in sepsis and in septic shock. We investigated this in a cohort of patients with sepsis by building a linear correlation model to determine the existence of a correlation between these two markers in sepsis and septic shock.

\section{Materials and Methods}

\subsection{Study population}

This was a retrospective study, carried out in the Fujian Medical University teaching hospital. The study was conducted according to the ethical principles for medical research stated in the Helsinki Declaration. The study was approved by the Ethics Committee of the Fujian Provincial Hospital.

Exclusion criteria included pregnancy and patients with advanced tumors or irreversible organ failure.

\subsection{Data collection}

Sepsis and septic shock were defined according to the Third International Consensus Definitions for Sepsis and Septic Shock (Sepsis-3) [17] Herein, sepsis is defined as life-threatening organ dysfunction caused by a dysregulated host response to infection. Patients with septic shock can be identified with a clinical construct of sepsis with persisting hypotension requiring vasopressors to maintain a mean arterial pressure (MAP) of $65 \mathrm{mmHg}$ and having a serum lactate level of $>2$ $\mathrm{mmol} / \mathrm{L}(18 \mathrm{mg} / \mathrm{dL})$ despite adequate volume resuscitation.

All patients were admitted to the intensive care unit (ICU). Within 1 hour of arrival, the subclavian vein was catheterised and blood samples were taken from the subclavian vein, as well as from the radial or femoral artery. Arterial blood lactic acid concentration and central venous oxygen saturation were determined using the GEM Premier 3500 system.

Patients were divided into the sepsis group (Group1_1) or 
TA B LE 1. Basic information of the study population.

\begin{tabular}{lcccc} 
& \multicolumn{2}{c}{ Group1 $(\mathrm{n}=37)$} & \multicolumn{2}{c}{ Group2 $(\mathrm{n}=37)$} \\
& No shock $(\mathrm{n}=17)$ & Shock $(\mathrm{n}=20)$ & Survival $(\mathrm{n}=26)$ & Death $(\mathrm{n}=11)$ \\
\hline Ages & $($ Group1_1) & $($ Group1_2) & $($ Group2_1) & (Group2_2) \\
\hline Sex & $64.88 \pm 12.55$ & $65.20 \pm 18.28$ & $69.15 \pm 12.25$ & $55.36 \pm 19.11$ \\
Chronic lung disease & Male:12(Female:5) & Male:16(Female:4) & Male:21 (Female:5) & Male:7 (Female:4) \\
Chronic heart disease & $5(29.4 \%)$ & $4(20 \%)$ & $8(30.8 \%)$ & $1(9.1 \%)$ \\
\hline Diabetes & $11(64.7 \%)$ & $9(45 \%)$ & $16(61.5 \%)$ & $4(36.3 \%)$ \\
Lung infection & $4(23.5 \%)$ & $6(30 \%)$ & $9(34.6 \%)$ & $1(9.1 \%)$ \\
\hline Abdominal infection & $7(41.2 \%)$ & $14(70 \%)$ & $16(61.5 \%)$ & $5(45.4 \%)$ \\
Blood system infection & $9(52.9 \%)$ & $4(23.5 \%)$ & $8(30.8 \%)$ & $5(45.5 \%)$ \\
\hline Urinary system infection & $1(5.9 \%)$ & $1(5 \%)$ & $1(3.8 \%)$ & $1(9.1 \%)$ \\
\hline
\end{tabular}

the septic shock group (Group1_2), according to the Sepsis3 definitions [17]. Patients were further divided according to outcome, into the survival (Group2_1) or the non-survival group (Group2_2).

\subsection{Statistical analyses}

Data analyses were conducted with SPSS 26 statistical software package (SPSS, Chicago, IL, USA).

First, we performed a missing value analysis of related variables, using the expectation-maximisation (EM) algorithm and regression. Where the Little's MCAR test did not reach significance $(\alpha=0.05)$, those missing values were replaced with the series mean.

Group differences with respect to lactate and $\mathrm{ScvO} 2$ were tested. Where data were normally distributed, a two-sample $\mathrm{t}$ test was carried out (with $\mathrm{p}<0.05$ representing statistical significance). Where data were non-normally distributed, nonparametric testing was carried out $(\mathrm{p}<0.05)$.

Correlation analysis was then conducted on the consecutive lactate and $\mathrm{ScvO} 2$ data, by the calculation of the Pearson correlation coefficient (with $\mathrm{p}<0.05$ representing statistical significance) and by linear regression analysis.

\section{Results}

37 patients within the sepsis population were included in this study. Patients were aged $29-86$ years (Table 1).

\subsection{Missing value analysis}

There were no missing values of lactate. Six values of $\mathrm{ScvO} 2$ were identified, with a deletion rate of $16.2 \%$. Statistical analysis of missing variables, by EM and regression, yielded a Little's MCAR test result of $p=0.799$, indicating that the missing value is missing completely at random. The series mean was used to replace these missing values.

\subsection{Differences between groups}

Data from the sepsis and septic shock groups were found to be non-normally distributed. Two independent sample nonparametric tests were carried out, with the finding that lactate concentrations were significantly lower in the sepsis group compared to the shock group ( $\mathrm{p}<0.05$, one-tailed). No differences of $\mathrm{ScvO}_{2}$ saturations were found between groups ( $p>0.05$, one-tailed) (Fig. 1).

Data from the survival and death groups did not conform to a normal distribution, and as such two independent sample nonparametric tests were carried out. Neither lactate nor $\mathrm{ScvO}_{2}$ levels were found to be significantly different between groups ( $p>0.05$, one-tailed) (Fig. 2).

\subsection{Linear correlation analysis}

Linear correlation analysis of all observed lactate and $\mathrm{ScvO}_{2}$ data yielded a Pearson correlation coefficient of $r^{2}=0.37(p<$ 0.001 ). Linear regression produced estimates for the slope and intercept of this linear relationship according to the equation $y$ $=-3.30 x+77.01$ (Fig. 3).

Similar analysis was conducted by subject group. In patients with sepsis, the correlation coefficient of lactate and $\mathrm{ScvO} 2$ was non-significant $\left(r^{2}=0, p>0.05\right)$. In the septic shock group, the correlation coefficient of lactate and $\mathrm{ScvO}_{2}$ was $\mathrm{r}^{2}$ $=0.46(\mathrm{p}=0.001)$. The linear equation was $y=-4.11 x+82.62$ (Fig. 4).

In the survival group, linear correlation analysis between lactate and $\mathrm{ScrO}_{2}$ was non-significant $\left(\mathrm{r}^{2}=010, \mathrm{p}>0.05\right)$. In the death group, the correlation coefficient of lactate and $\mathrm{ScvO}_{2}$ was $\mathrm{r}^{2}=0.67(\mathrm{p}<0.01)$. The linear equation was $y=$ $-3.70 x+78.61$ (Fig. 5).

\section{Discussion}

Persistently improving of lactate levels suggests ongoing inadequacy of oxygen delivery. High levels of $\mathrm{ScvO}_{2}$ indicate the impaired of the cellular oxygen utilization and microcirculatory, which suggest a systemic oxygen delivery in excess of oxygen demand. Low levels of $\mathrm{ScvO}_{2}$ suggest inadequate oxygen delivery for metabolic demands.

Lactate, which is the product of anaerobic metabolism, is considered the biomarker of choice for reflecting the presence of tissue hypoxia $[18,19]$. Reduction in $\mathrm{ScvO}_{2}$ occurs when oxygen delivery to tissues is reduced and oxygen extraction is increased [20]. Elevated $\mathrm{ScvO}_{2}$ suggests a systemic oxygen 

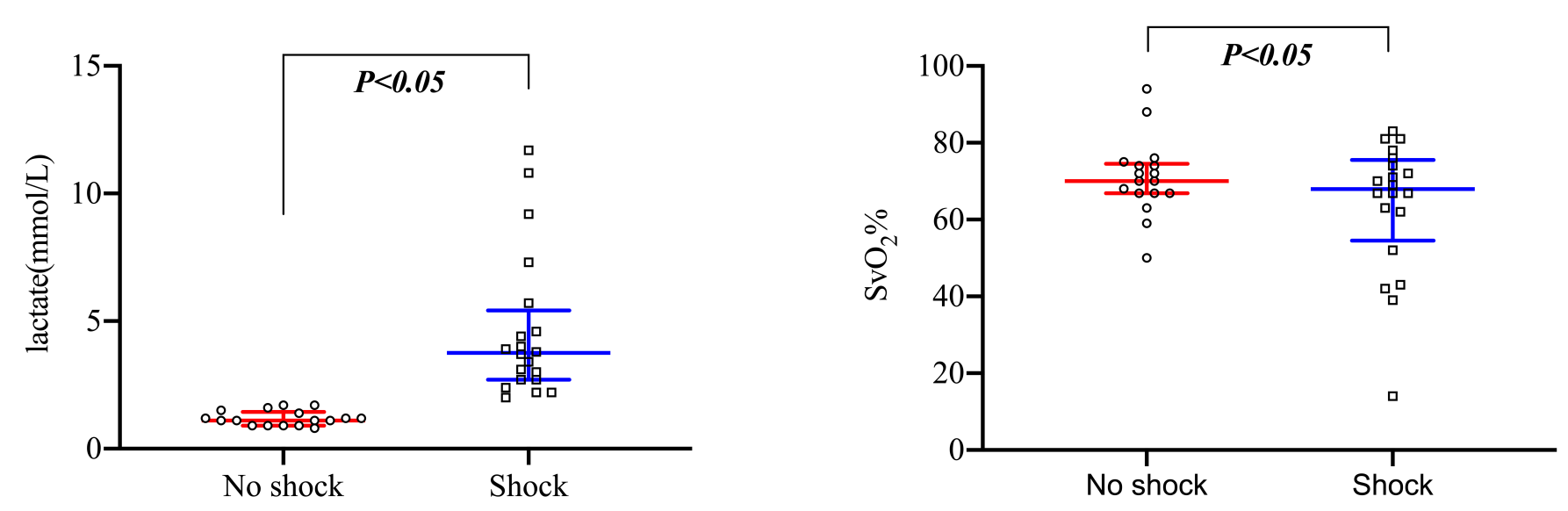

F I G U R E 1. Comparison of lactate and $\mathrm{ScvO}_{2}$ between the sepsis group and septic shock group.
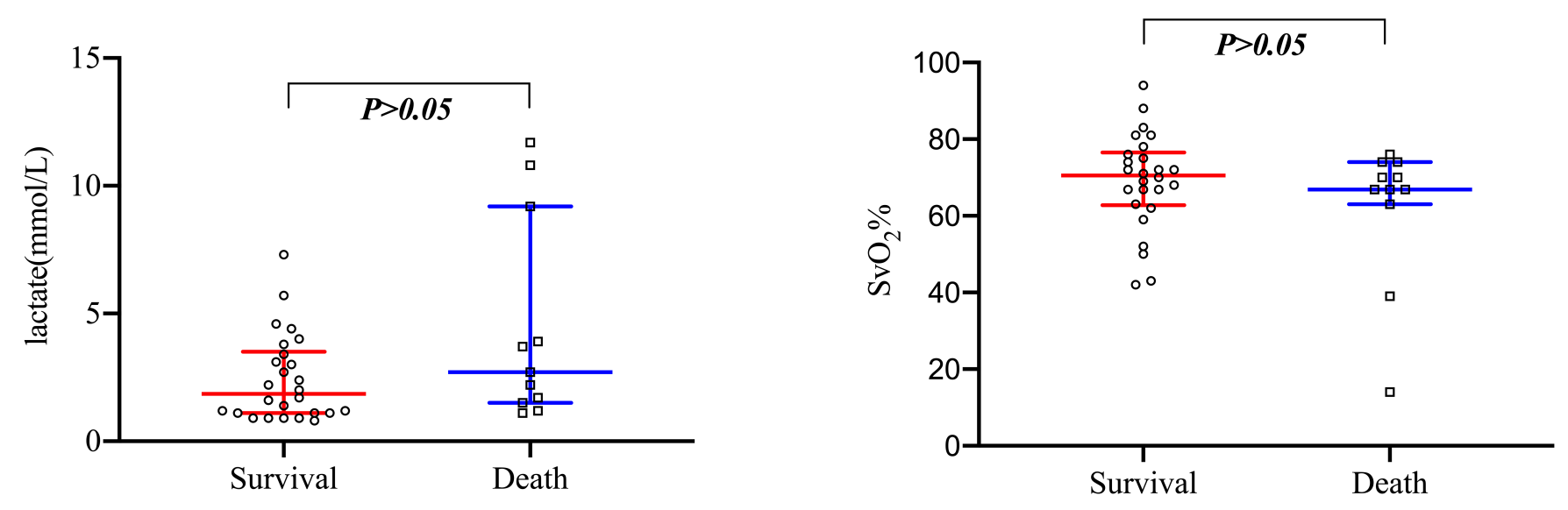

F I G U RE 2. Comparison of lactate and $\mathrm{ScvO}_{2}$ between the survival group and death group.

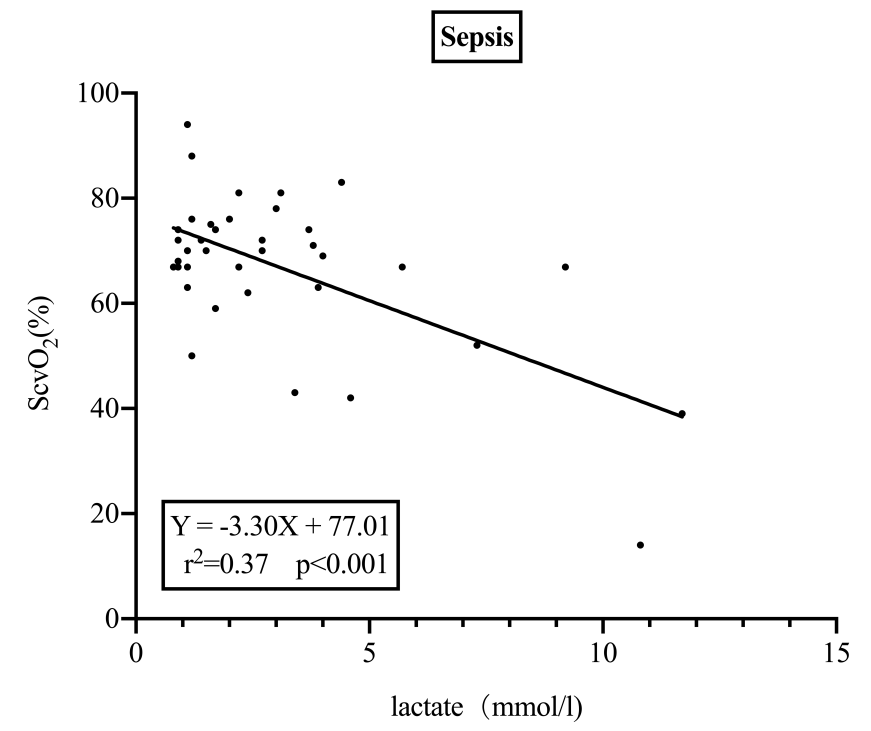

FIGURE 3. Correlation analysis between lactate and $\mathrm{ScvO}_{2}$ in the sepsis population.

delivery in excess of oxygen demand, impaired mitochondrial oxygen utilization, and/or microcirculatory shunting. Low $\mathrm{ScvO}_{2}$ values imply inadequate oxygen delivery that fails to meet metabolic demands [21].

Early goal-irected therapy (EGDT) for patients with sepsis and septic shock was first implemented by Rivers et al., based on a landmark single center randomized controlled clinical trial (RCT) in which a $16 \%$ mortality reduction was achieved by treatment targeting of $\mathrm{ScvO}_{2}, \mathrm{MAP}$, central venous pressure and urine output within the first 6 hours after diagnosis [22]. More recently, three multicenter RCTs showed that EGDT did not confer survival benefit compared with usual care for patients with sepsis and/or septic shock, along with suggestions that it should be excluded from the guideline [23, 24]. Interestingly, none of these trials addressed the utility of $\mathrm{ScvO}_{2}$ as a target for resuscitation of septic shock [12], because half of the included patients had normal $\mathrm{ScvO}_{2}$ at the time of randomization. However, studies have shown that lactate and $\mathrm{ScvO}_{2}$ are independent predictors of mortality [25] and that normalization of either or both biomarkers is associated with improved outcomes in sepsis and septic shock $[19,26]$. This is consistent with our findings, which demonstrate a complex relationship between lactate and $\mathrm{ScvO} 2$. In the implementation of $\mathrm{ScvO} 2$ to assess the balance between tissue oxygen supply and consumption in sepsis, the choice of time point is crucial [11].

The present study is an analysis of the relationship between 

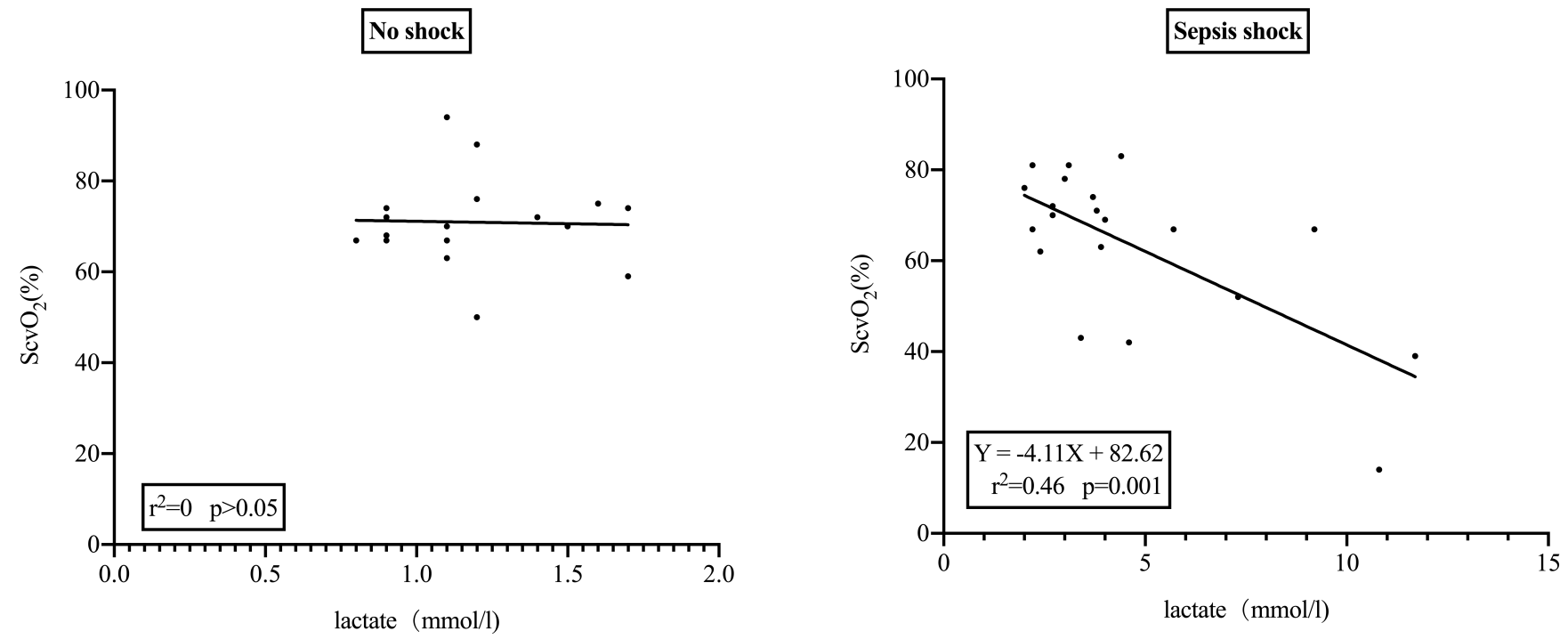

F I G U RE 4. Correlation analysis between lactate and $\mathrm{ScvO}_{2}$ in the sepsis and septic shock groups.
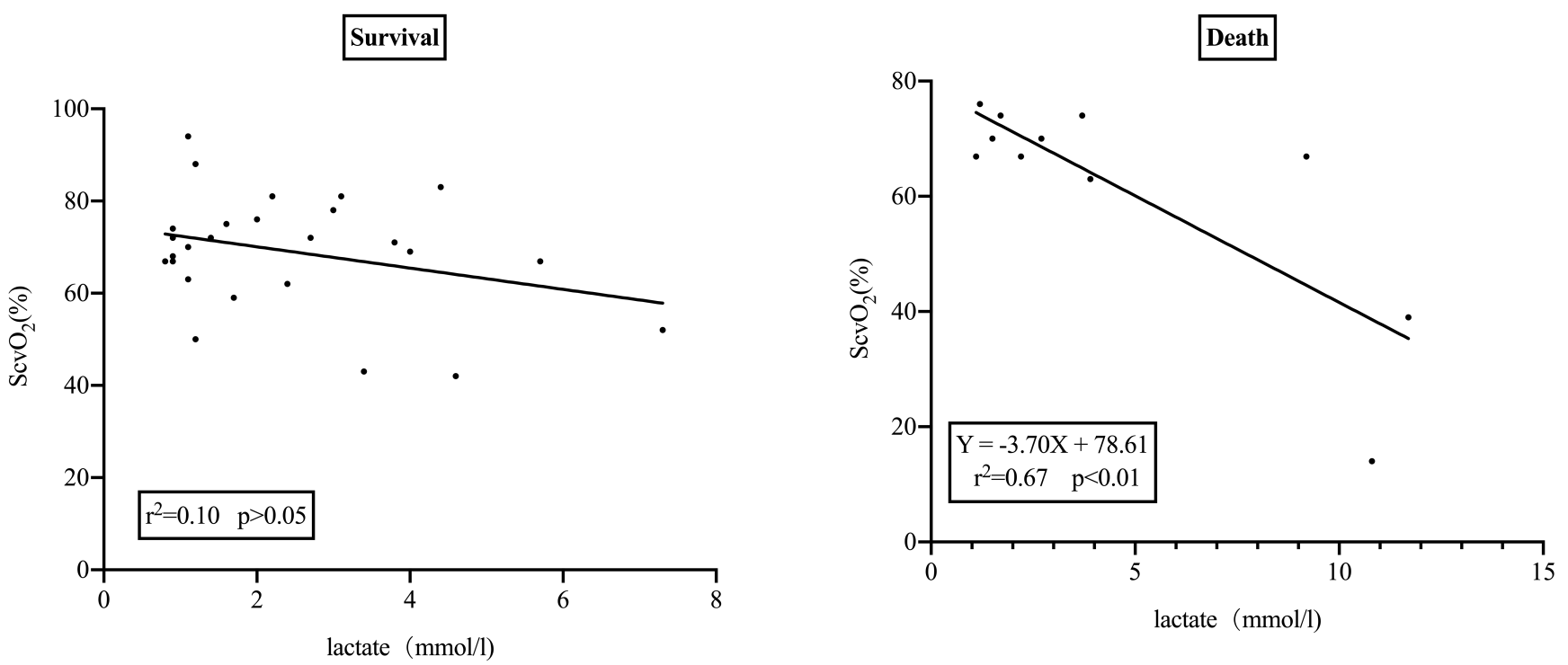

F I G U RE 5. Correlation analysis between lactate and $\mathrm{ScvO}_{2}$ in the survival group and death group.

lactate and $\mathrm{ScvO} 2$ in sepsis patients. To our knowledge it is the first analysis of these two markers after stratifying sepsis cases by severity. This study had several limitations, however. First, it is a single-center study including relatively few patients. Second, this study was cross-sectional, so its ability to infer causality is limited.

\section{Conclusion}

In sepsis, the correlation between $\mathrm{ScvO}_{2}$ and lactate is not constant. As the disease progresses, the correlation may be dynamic. Therefore, in the resuscitation of sepsis and/or septic shock, changes in central venous blood oxygen saturation need attention.

\section{ACKNOWLEDGMENTS}

We would like to thank the participants for providing the information used in this study and for kindly making the arrangements for data collection.

\section{CONFLICT OF INTEREST}

The authors declare no conflicts of interest relevant to this article.

\section{LIST OF ABBREVIATIONS}

$\mathrm{ScvO}_{2}$ : Central Venous Oxygen Saturation; MAP: Mean Arterial Pressure; ICU: Intensive Care Unit; EGDT: Early Goal Directed Therapy; RCT: Randomized Controlled Clinical Trial 


\section{ETHICAL APPROVAL}

The study has been approved by the Fujian Provincial Hospital Ethics Committee.

\section{AVAILABILITY OF DATA AND MATERIALS}

The datasets used and/or analysed during the current study are available from the corresponding author on reasonable request.

\section{FUNDING}

This study was supported by the Natural Science Foundation of Fujian Province (Grant No. 2019J01175), and the Young and Middle-aged Talents Training Project of Fujian Provincial Health Commission (Grant No. 2018-ZQN-1).

\section{AUTHORS' CONTRIBUTIONS}

Songchang Shi and Wei Lin performed the statistical analysis and were the major contributors in writing the manuscript. Xiaobin Pan, Chao Wu and Mei Ye interpreted the data. Songjing Shi and Xingsheng Lin reviewed and designed the study. All authors read and approved the final manuscript. Songjing Shi is the guarantor of this manuscript and, as such, had full access to all the data in the study and takes responsibility for the integrity of the data and the accuracy of the data analysis.

\section{REFERENCES}

[1] Angus DC, Linde-Zwirble WT, Lidicker J, et al. Epidemiology of severe sepsis in the United States: Analysis of incidence, outcome, and associated costs of care. Crit Care Med. 2001;29:1303-1310.

[2] Lin W, Huang H, Wen J, et al. The predictive value of procalcitonin for early detection of infection in elderly type 2 diabetes mellitus. J Infect Chemother. 2020;26:343-348.

[3] Vincent J-L, Marshall JC, Namendys-Silva SA, et al. Assessment of the worldwide burden of critical illness: the Intensive Care Over Nations (ICON) audit. Lancet Respir Med. 2014;2:380-386.

[4] Fleischmann C, Scherag A, Adhikari NKJ, et al. Assessment of Global Incidence and Mortality of Hospital-treated Sepsis. Current Estimates and Limitations. Am J Respir Crit Care Med. 2016;193:259-272.

[5] Jawad I, Lukšić I, Rafnsson SB. Assessing available information on the burden of sepsis: global estimates of incidence, prevalence and mortality. J Glob Health. 2012;2:010404.

[6] Annane D, Bellissant E, Cavaillon J-M. Septic shock. Lancet. 2005;365:63-78.

[7] Rhee C, Dantes R, Epstein L, et al. Incidence and Trends of Sepsis in US Hospitals Using Clinical vs Claims Data, 2009-2014. JAMA. 2017;318:1241.

[8] Weisberg A, Park P, Cherry-Bukowiec JR. Early Goal-Directed Therapy:
The History and Ongoing Impact on Management of Severe Sepsis and Septic Shock. Surg Infect (Larchmt). 2018;19:142-146.

[9] Bakker J, Nijsten MWN, Jansen TC. Clinical use of lactate monitoring in critically ill patients. Ann Intensive Care. 2013;3:12.

[10] Walley KR. Use of Central Venous Oxygen Saturation to Guide Therapy. Am J Respir Crit Care Med. 2011;184:514-520.

[11] Gattinoni L, Vasques F, Camporota L, et al. Understanding Lactatemia in Human Sepsis. Potential Impact for Early Management. Am J Respir Crit Care Med. 2019;200:582-589.

[12] Bisarya R, Shaath D, Pirzad A, et al. Serum lactate poorly predicts central venous oxygen saturation in critically ill patients: a retrospective cohort study. J Intensive Care. 2019;7:47.

[13] Dickstein K, Cohen-Solal A, Filippatos G, et al. ESC Guidelines for the diagnosis and treatment of acute and chronic heart failure 2008. Eur J Heart Fail. 2008;10:933-989.

[14] Gallet R, Lellouche N, Mitchell-Heggs L, et al. Prognosis value of central venous oxygen saturation in acute decompensated heart failure. Arch Cardiovasc Dis. 2012;105:5-12.

[15] Lazzeri C, Valente S, Chiostri M, et al. Clinical significance of Lactate in acute cardiac patients. World J Cardiol. 2015;7:483.

[16] NÉMETH M, TÁNCZOS K, DEMETER G, et al. Central venous oxygen saturation and carbon dioxide gap as resuscitation targets in a hemorrhagic shock. Acta Anaesthesiol Scand. 2014;58:611-619.

[17] Singer M, Deutschman CS, Seymour CW, et al. The Third International Consensus Definitions for Sepsis and Septic Shock (Sepsis-3). JAMA. 2016;315:801.

[18] Bakker J, Nijsten MWN, Jansen TC. Clinical use of lactate monitoring in critically ill patients. Ann Intensive Care. 2013;3:12.

[19] Jones AE. Lactate clearance vs central venous oxygen saturation as goals of early sepsis therapy: a randomized clinical trial. JAMA. 2010;303:739746.

[20] Walley KR. Use of central venous oxygen saturation to guide therapy. Am J Respir Crit Care Med. 2011;184:514-520.

[21] Semler MW, Singer M. Deconstructing Hyperlactatemia in Sepsis Using Central Venous Oxygen Saturation and Base Deficit. Am J Respir Crit Care Med. 2019;200:526-527.

[22] Liu B, Ding X, Yang J. Effect of early goal directed therapy in the treatment of severe sepsis and/or septic shock. Curr Med Res Opin. 2016;32:1773-1782.

[23] Peake SL, Delaney A, Bailey M, et al. Goal-Directed Resuscitation for Patients with Early Septic Shock. N Engl J Med. 2014;371:1496-1506.

[24] Mouncey PR, Osborn TM, Power GS, et al. Trial of Early, Goal-Directed Resuscitation for Septic Shock. N Engl J Med. 2015;372:1301-1311.

[25] Shin TG, Jo IJ, Hwang SY, et al. Comprehensive Interpretation of Central Venous Oxygen Saturation and Blood Lactate Levels During Resuscitation of Patients With Severe Sepsis and Septic Shock in the Emergency Department. Shock. 2016;45:4-9.

[26] Filho RR, Rocha LL, Corrêa TD, et al. Blood Lactate Levels Cutoff and Mortality Prediction in Sepsis-Time for a Reappraisal? a Retrospective Cohort Study. SHOCK. 2016;46:480-485.

How to cite this article: Songchang Shi, Xiaobin Pan, Wei Lin, Chao Wu, Mei Ye, Yingfeng Zhuang, et al. Establishment of a Linear Correlation Model of Central Venous Blood Oxygen Saturation and Lactate in Sepsis. Signa Vitae. 2021;17(1):101-105. doi:10.22514/sv.2020.16.0064. 